\title{
Automating Energy Management in Green Homes
}

\author{
Nilanjan Banerjee* \\ University of Arkansas \\ Fayetteville, AR, USA \\ nilanb@uark.edu
}

\author{
Sami Rollins* \\ University of San Francisco \\ San Francisco, CA, USA \\ srollins@cs.usfca.edu
}

\author{
Kevin Moran \\ University of San Francisco \\ San Francisco, CA, USA \\ ksmoran@cs.usfca.edu
}

\begin{abstract}
Homes powered fully or partially by renewable sources such as solar are becoming more widely adopted, however energy management strategies in these environments are lacking. This paper presents the first results of a study that explores home automation techniques for achieving better utilization of energy generated by renewable technologies. First, using a network of off-the-shelf sensing devices, we observe that energy generation and consumption in an off-grid home is both variable and predictable. Moreover, we find that reactive energy management techniques are insufficient to prevent critical battery situations. We then present a recommendation based system for helping users to achieve better utilization of resources. Our study demonstrates the feasibility of three recommendation components: an early warning system that allows users of renewable technologies to make more conservative decisions when energy harvested is predicted to be low; a task rescheduling system that advises users when high-power appliances such as clothes dryers should be run to optimize overall energy utilization; and an energy conservation system that identifies sources of energy waste and recommends more conservative usage.
\end{abstract}

\section{Categories and Subject Descriptors}

H.1.2 [Models and Principles]: User/Machine Systemshuman factors

\section{General Terms}

Human Factors

\section{INTRODUCTION}

Homes that derive part or all of their energy from renewable sources, such as solar and wind, are becoming more widely adopted. Though early adopters of renewable technologies

\footnotetext{
*lead co-authors listed in alphabetical order
}

Permission to make digital or hard copies of all or part of this work for personal or classroom use is granted without fee provided that copies are not made or distributed for profit or commercial advantage and that copies bear this notice and the full citation on the first page. To copy otherwise, to republish, to post on servers or to redistribute to lists, requires prior specific permission and/or a fee.

HomeNets'11, August 15, 2011, Toronto, Ontario, Canada.

Copyright 2011 ACM 978-1-4503-0798-7/11/08 ...\$10.00. often report that their motivation for adoption was a desire to be more environmentally responsible, new pricing models such as leases have made renewables more affordable. As a result, renewables have become an alternative for people interested in reducing their energy costs. In the US, the number of grid-tied homes with photovoltaic installations grew by $40 \%$ in $2009[8,16]$. The growth was largely fueled by decreasing cost of solar technologies and increase in incentives offered to consumers who install the technology. These trends are unlikely to reverse [16].

Technology and services that help consumers to manage the energy generated by their renewables have improved in recent years. We spoke to one user who installed solar panels at his home in 2002 and who, in order to log the performance of the system, manually recorded readings from a control panel on a daily basis over a period of several months. Another user, however, who is in the process of installing solar panels, reports that he will have access to a web-based service that reports overall energy generation and consumption at his home. There has also been a significant increase in technologies, such as the WattsUpMeter [12] and Android@Home [1], that allow users to monitor the fine-grained power consumption of individual appliances, as well as services, such as Google PowerMeter $[4,7,10]$, that provide users with convenient access to such information. These technologies, however, do little more than provide access to raw data. The user must still attentively monitor readings, aggregate the information collected by a diverse set of sensing devices, and decide how to use the information to make smart decisions about energy consumption.

This paper presents the first results of a study that explores automated techniques for changing energy consumption patterns to achieve better utilization of available resources in green homes. Through an empirical case study, we derive four key insights that demonstrate the need for and the feasibility of automated energy management in green homes. First, we explore how energy is both generated and consumed in an off-grid home entirely powered by solar panels. Using a network of off-the-shelf sensing devices, we observe that energy generation and consumption in the home is both variable and predictable. Moreover, we observe that the residents in the home use reactive energy management techniques - they wait until the energy supply is low before taking action to reduce overall energy consumption. This approach, we find, is not sufficient to prevent the critical situation in which a generator must be used to power the home. Based on our observations, we propose a recommendation-based system that monitors energy harvested and consumed and provides 


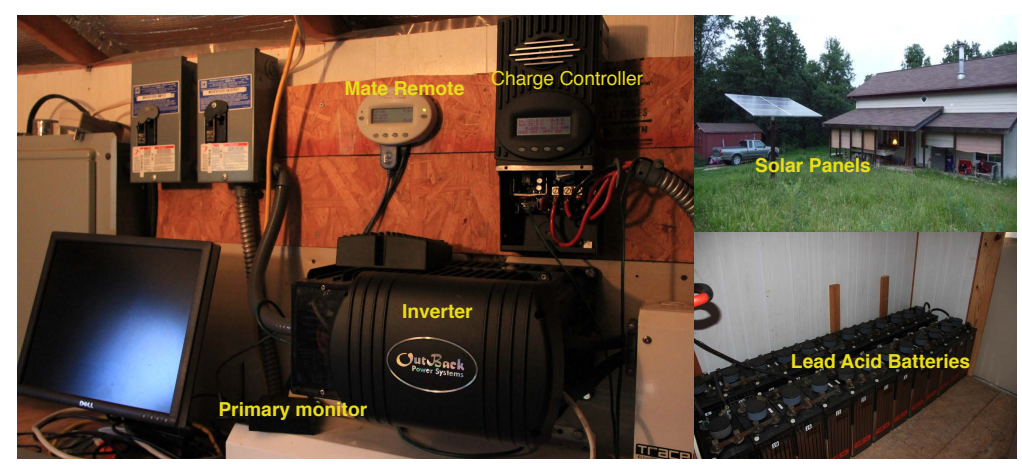

Figure 1: The monitoring setup at the off-grid, solar-powered home. The primary monitoring device is a FitPC tethered to a Mate remote control that profiles the inverter. It is possible to access the solar charging and energy expended data over a standard RS232 interface using custom software.

users with feedback and advice on how to adjust energy consumption patterns via a smartphone application. Our study demonstrates the feasibility of three recommendation components: an early warning system that allows users of renewable technologies to make more conservative decisions when energy harvested is predicted to be low; a task rescheduling system that advises users when high-power appliances such as clothes dryers should be run to optimize overall energy utilization; and an energy conservation system that identifies sources of energy waste and recommends more conservative usage.

This exploratory study focuses on an off-grid, solar home in Arkansas. We recognize that off-grid users are an extremely small segment of the population, and our goal is not to develop technology that will only be useful in an off-grid home. There are two significant benefits to working in this space, however. First, these users are at the extreme end of the spectrum when it comes to conservation. Understanding and classifying the manual techniques they have developed for demand-response can inform the recommendations we can make to other grid-tied, or even standard grid users. Second, off-grid users are likely to be enthusiastic early adopters of our technology, helping us to validate and improve our techniques without a significant barrier to adoption.

\section{AN OFF-GRID SOLAR HOME}

To understand the semantics governing energy utilization in green homes, we have deployed a suite of monitoring tools in a solar-powered home in Fayetteville, Arkansas. The goal of the monitoring infrastructure is to drive measurement-driven analysis to understand how users in solar-powered houses interact with energy. The house is occupied by a couple; one occupant works for the University of Arkansas and the other occupies the house most of the day.

Our deployment is shown in Figure 1. The house is located several kilometers from the city and is powered by 8 Kyocera $120 \mathrm{~W}$ solar panels [11]. The solar panels drive $242 \mathrm{~V} 840$ Ah Lead Acid batteries (two banks of 12 batteries $=24 \mathrm{~V}$ ) through a FlexMax 60 charge controller [13]. A VFX3524 Power Inverter is used to transform DC current to AC, which powers the appliances at home. We have augmented the above setup with a monitoring station that consists of a low-power FitPC [6]. A custom software tool on the FitPC periodically collects data from a controller device (called the MATE remote [14]) that collects data from the inverter.
Data at the rate of one sample per minute is captured over the RS232 interface and written to an append-only log. Each sample consists of the instantaneous residual battery voltage (in Volts) and the energy consumed by the house (in KWh). The amount of energy scavenged from the panels can be calculated using these values and the maximum battery capacity. At this time the log files are manually transferred by the house occupants. In addition to monitoring the cumulative energy consumption for the home, we have deployed networked WattsUpMeters to monitor individual appliances. We currently monitor a television and a refrigerator. We have collected data over a period of 55 days (14 days during the summer of 2010 and 41 days in Nov-Dec 2010) and plan to continue collecting data over the next two years.

\section{DATA ANALYSIS}

Analysis of the data collected by our monitoring infrastructure highlights four key findings that have informed the design of our automated energy management system.

Traditional energy management techniques are insufficient in off-grid homes.

First, we consider whether established norms of energy management, for example the 7PM-7AM policy that recommends users run important household appliances between $7 \mathrm{PM}$ and 7AM when the demand for power is low, are appropriate for green homes. Figure 2 shows the total energy consumption in the off-grid house at different times of the day over 41 winter days. While the energy consumption varies considerably over 24 hours, we find that a large portion of energy is consumed between 10AM-8PM (10-20 on the $\mathrm{x}$-axis). Conversations with our subject indicate that consumption is primarily driven by residual energy in their batteries. During the day when the batteries are close to full, the users maximally use their household appliances like washers and water heater. This is, in fact, the opposite of the 7PM-7AM strategy. These findings demonstrate a need to develop new energy management techniques that consider the unique energy harvesting conditions in homes powered by renewable technologies. 


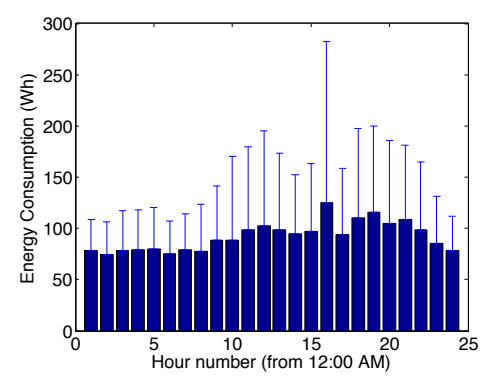

Figure 2: Energy consumption during different hours of the day averaged over 41 winter days. The errorbars indicate the standard deviation across 41 days.

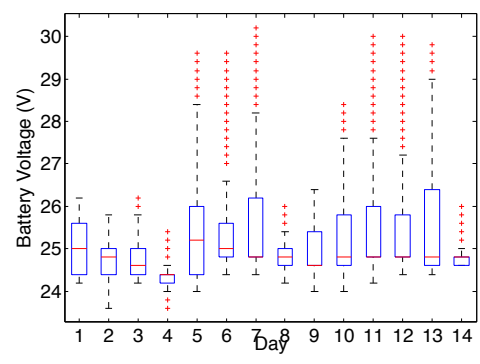

Figure 3: Variability in battery voltage in the off-grid home across a single day over 14 summer days in 2010.

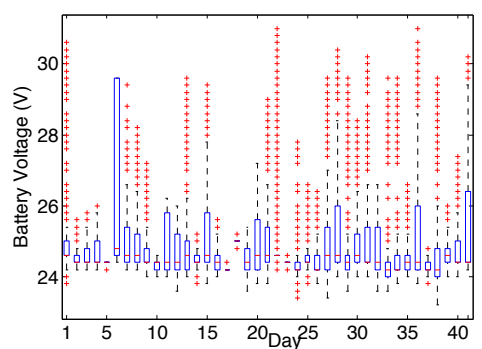

Figure 4: Variability in battery voltage in the off-grid home across a single day over 41 winter days in 2010.
Variability in energy generation and consumption suggests the need for adaptive management strategies.

We next consider whether a simple, fixed strategy for energy management would be appropriate for off-grid homes. Figure 3 and Figure 4 are box-plots of the battery voltage (in the lead-acid batteries in the off-grid home) over 14 summer and 41 winter days in 2010. Each point on the x-axis corresponds to data collected over a single day. The battery voltage is a function of the energy harvested from the panels and energy consumed by the house. Although the overall energy consumed per day, shown in Figure 5 varies, a large proportion of the variability in the battery voltage can be attributed to the energy harvested from the panels. From the figure, we conclude that energy harvested from the panels and energy consumed by the house are both highly variable. There is variance across a single day, across several days, and across seasons. This suggests that fixed energy management strategies are insufficient and adapting to variability is a key element for green homes.

\section{Reactive techniques do not prevent critical battery situations.}

We further analyze whether manual techniques employed by our green home user are sufficient. Our user reports that he is extremely conservative; he monitors the voltage of his batteries and reduces consumption, for example by switching off lights, when the voltage drops below $90 \%$. Even so, there are periods of time when he must use a generator to power his home. We observed that he used the generator on 10 days during the 41-day winter data collection period-approximately $25 \%$ of the time. To understand what influenced the use of the generator, in Figure 10 we plot the battery voltage over 24 hours for three instances before the generator was used. It is clear that the period saw a gradual decrease in the voltage and the $\mathrm{AC}$ generator was used when the residual battery voltage was close to $24 \mathrm{~V}$. Our conversation with our subject further ratified the above observation. While he agreed that most of the time the AC generator was used when the battery capacity was low, on one occasion he had used the generator to "equalize" the batteries - a process of overcharging the batteries for a few hours which causes boiling of the electrolyte (acid) and helps breakup any calcification on the lead plates and stratification of the acid ${ }^{1}$. To understand whether our subject used any techniques to prevent the use of the generator, we plot the total energy consumption of the house over 24 hours before the generator was used in Figure 6 . Although there is a decrease in energy consumption in the last 10 hours, it did not circumvent the use of the generator. Hence, manual reactive techniques to prevent energy outages are not sufficient to prevent the system from running out of charge.

\section{Energy generation and consumption exhibit predictabil- ity.}

Finally, we consider whether energy generation and consumption patterns in green homes can be predicted in order to inform optimal energy management decisions. To quantify the predictability of the harvester source and energy consumed by appliances, in Figure 7 we calculate correlograms (autocorrelation at different lags) from the battery voltage data and energy consumed by the television set in our solar home. A lag corresponds to one hour. Hence, if the autocorrelation at lag $k$ is $a_{k}$, it implies that the correlation between data at time $t$ and $t+k$ hours is $a_{k}$. For battery voltage, the autocorrelation decreases with increasing lag, hence the battery capacity is predictable at short time spans - for example, around every 5-10 hours. However, the television data shows high correlation at short lags and lags around 20 hours. The higher correlation at 20 hours is due to the periodic use of the TV set. Our subject watches the television (located in his kitchen) when he cooks in the evening. Therefore, while there is variability in energy consumed and generated, there is considerable predictability in the data, pointing to the feasibility of automated and proactive energy management schemes

\section{SYSTEM DESIGN}

Our measurement analysis demonstrates both the need for and the viability of energy management techniques that consider the unique properties of renewable energy sources. We propose a system that exploits these properties and offers the following contributions:

\footnotetext{
${ }^{1}$ We found that on one instance (not shown in the figure) the generator was used when the voltage was close to $29 \mathrm{~V}$
} 


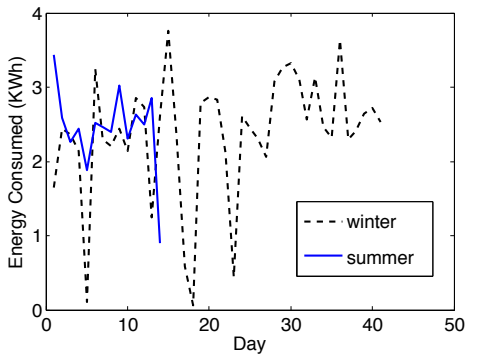

Figure 5: Total energy consumption of the off-grid home during 14 summer and 41 winter days.

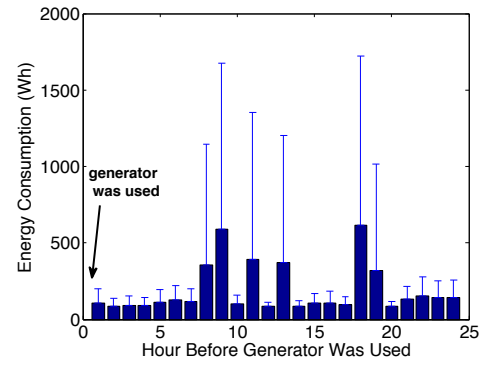

Figure 6: Energy consumption during 24 hours before the AC generator was used. The errorbars indicate the standard deviation over 10 different instances.

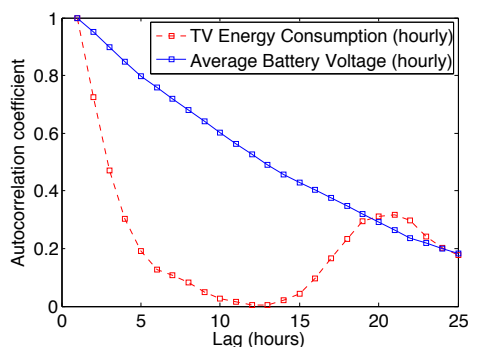

Figure 7: Autocorrelation at different lags (a lag equal to an hour) for the battery voltage data and energy consumed by a TV set.
- Early Warning: By predicting times when a home's energy store is likely to be critically low, the user can be notified in advance and take proactive measures for reducing energy consumption. This both saves the user from the manual task of monitoring the state of the home's batteries and it also allows for earlier and more accurate detection of critical situations.

- Task Rescheduling: By predicting when energy harvesting is at its peak or when energy storage is full, the user can be advised of the best times to execute highpower tasks such as running a dishwasher or clothes washer.

- Energy Conservation: Even the manual energy conservation techniques employed by our extremely conservative off-grid user can be improved. For each device from which we collect power measurements, we identify a risk [9] - the margin of error that is tolerable by the user. We use this information to suggest more conservative power states, for example adjusting the temperature of a refrigerator up one or two degrees, that will not have a noticeable impact for the user.

Though our data demonstrates the utility of these recommendations in an off-grid environment, our goal is to design a system that will meet the needs of green home users in both off-grid and grid-tied homes. While an off-grid user may want to maximally use power during peak generation times, a grid-tied user may actually want to minimize power use and maximize revenue by selling power back to the grid during peak times. By identifying peak and low generation times in advance, our system allows a user to decide to maximally or minimally use available energy to meet the desired objective.

We present a recommendation-based system that monitors energy generation and consumption and provides users with advice, including warnings in advance of critical battery situations, recommendations for the best times to execute highpower tasks, and opportunities to adjust the power states of devices to reduce energy consumption. We have chosen a recommendation-based model to minimize user irritation and ensure that control of household appliances ultimately resides with the user. A smartphone application notifies the user when the system suggests changes to the power states of devices, for example suggests that the user turn an appliance on. The user is responsible for implementing the suggestion, and control of the devices is supported via the smartphone application. Figure 8 illustrates the primary components of the system.

Monitoring Infrastructure: The monitoring infrastructure collects raw data on energy generation and consumption. Minimally, the system requires measurements of the energy generated by a renewable source and the overall energy consumption of the house. Our system, however, can be improved by integrating fine-grained power measurements of individual appliances.

Web Application and Scheduling Logic: A central server implements the logic of an optimization algorithm and exposes recommendations via a web service accessed by a smartphone application. A profiler aggregates data collected by all components of the monitoring infrastructure and provides the data to a scheduler. The scheduler implements an optimization framework that determines appropriate recommendations. Our present optimization framework (work-in-progress) allocates energy from batteries to appliances proportional to their consumption in the past and their associated risk. When a new recommendation is available, the scheduler notifies the web service, which pushes a notification to an iPhone application. The iPhone application can then retrieve further information, including specifics about the recommendation or raw data produced by the monitoring infrastructure, for example the current power draw of a specific appliance.

Control System: The smartphone application, shown in Figure 9, both displays the recommendations and other information about the status of devices in the home and provides the user with the ability to control supported devices from the phone itself. Commands issued in the application, for example adjusting the brightness of a lamp, generate requests to the web service. The web service forwards requests to a control infrastructure that can directly control individual devices. The control infrastructure is an application built on top of the HomeOS $[3,5]$ framework that provides the necessary drivers and API to control appliances remotely. 


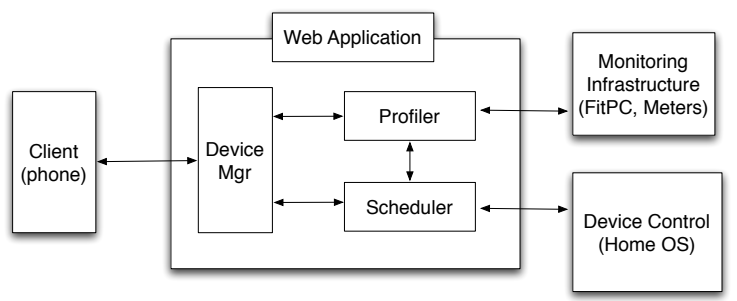

Figure 8: The design of a recommendationbased system for improving energy utilization in a green home.

\section{FEASIBILITY ANALYSIS}

We are currently in the process of testing our components and deploying our recommendation system in the off-grid home. In this section, we demonstrate the feasibility of generating useful recommendations.

Battery voltage can be used to predict when the generator will be necessary.

The early warning system requires accurate predictions of when the AC generator is likely to be used. Moreover, it is critical that the prediction occurs long before the actual event so that remedial actions can be taken. Figure 10 shows the battery voltage over 24 hours for three instances before the generator was used. The trend shows that the generator is used when the voltage either remains close to $24 \mathrm{~V}$ for a long time, or has a gradual decrease from a higher voltage to $24 \mathrm{~V}$. Such a trend can be captured using simple autoregressive or moving average models [2], demonstrating the feasibility of predicting energy outages long before they occur.

There are instances when the battery voltage is high and overall energy consumption is low.

To demonstrate that task rescheduling can help conserve energy in green homes, we present a scatter plot of battery voltage and overall energy consumption of the house in Figure 11. Each point in the plot corresponds to the energy consumed and the average battery voltage during a particular hour. The figure shows that there are several instances when the battery voltage is low (the AC generator is likely to be used) and the energy consumption is high. It also demonstrates that it is frequently the case that the voltage is high and energy consumption is low. Such a mismatch points to the potential for rescheduling delay-tolerant tasks, such as running a clothes washer, such that they occur when there is significant capacity in the batteries that is not being used for other critical tasks.

Even our conservative off-grid user could reduce energy usage.

Finally to corroborate the feasibility of energy conservation using risk associated with appliances, we present results from an experiment using a television set in our solar house in Arkansas. Figure 12 shows the power draw of the TV set during two instances of its use. We observe that on both
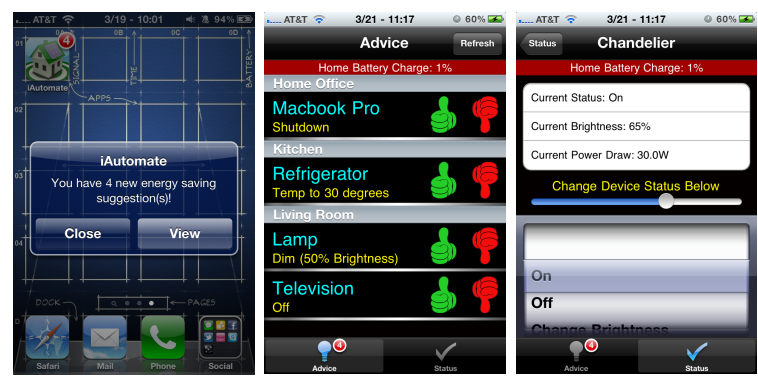

Figure 9: A smartphone application that displays system recommendations, collects user feedback, and allows control of selected appliances.

occasions, the power draw can be reduced by $3 \mathrm{~W}$ (quantified as risk) without affecting the user.

\section{FUTURE WORK}

This paper explores the feasibility of using automated techniques for energy management in homes powered by renewable technologies. The data collected by a network of off-the-shelf components deployed in a single off-grid home demonstrates the potential for a system that changes energy consumption patterns to achieve better utilization of available resources. Our current work focuses on the following areas:

- Improved Monitoring: We are developing auxiliary monitoring devices that will supplement the data collected by the off-the-shelf components with information about environmental conditions, such as ambient light, temperature, and humidity, that have a causal relationship with energy consumption (similar to $[15,17]$ ). This will help us to derive correlations between energy consumed and ambient environmental conditions and will provide insight on how humans duty cycle appliances based on environmental conditions. There are several challenges to overcome, however, including how to collect data in a non-intrusive way.

- Holistic System: We are building a general-purpose optimization framework that will adaptively balance the amount of energy harvested with energy expended such that usability needs are met. This requires identifying the importance of individual devices and allocating the energy predicted to be harvested accordingly.

- User Evaluation: We plan to deploy the web and iPhone application to collect information about the utility of the recommendations generated by the scheduling algorithm. The system will collect in situ feedback about whether recommendations are useful and correlate the recommendations with the power readings from individual devices.

- Wider Deployment: We are in the process of identifying other users, in off-grid and grid-tied homes that are only partially powered by renewable sources, who are willing to participate in our study. 


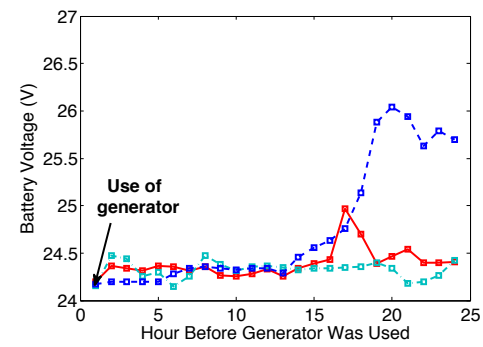

Figure 10: Battery voltage over 24 hours for three instances before the AC generator was used. Voltages drop from a high or are consistently low before the generator is used.

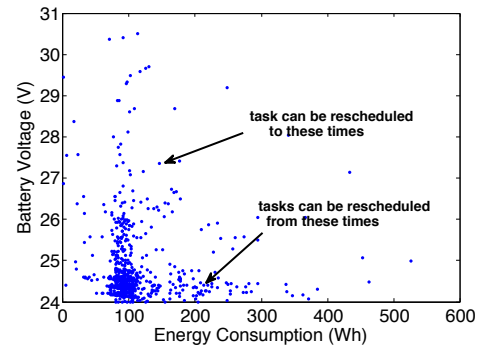

Figure 11: Scatter plot showing instances when the energy consumption and battery voltage are inversely correlated. Rescheduling tasks can help conservation.

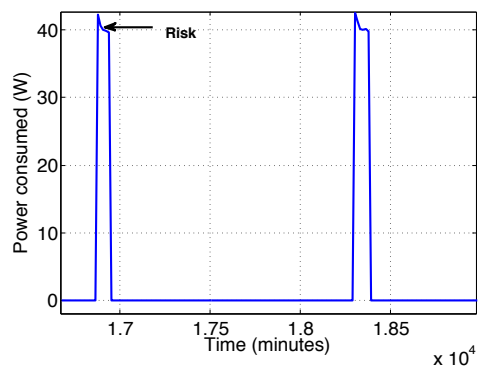

Figure 12: Risk associated with a television set- $3 \mathrm{~W}$ of power can be reduced without affecting the user.

\section{Acknowledgments}

We are thankful to the anonymous reviewers for their insightful comments and suggestions. We would also like to thank Ratul Mahajan for sharing the HomeOS platform executables with us and our off-grid house subject for volunteering to have his home monitored. This work was partially funded by National Science Foundation grants CNS-1018112 and CNS-1055061. Any opinions, findings, and conclusions or recommendations expressed in this material are those of the authors and do not necessarily reflect the views of the National Science Foundation.

\section{REFERENCES}

[1] Android @ Home.http://developer.android.com/ guide/topics/usb/adk.html.

[2] Autoregressive moving-average models. http://en.wikipedia.org/wiki/Autoregressive_ moving_average_model.

[3] A. B. Brush, B. Lee, R. Mahajan, S. Agarwal, S. Saroiu, and C. Dixon. Home Automation in the Wild: Challenges and Opportunities. In ACM CHI, 2011.

[4] Cisco Home Energy Management. http: //www.cisco.com/web/consumer/products/hem.html.

[5] C. Dixon, R. Mahajan, S. Agarwal, A. Brush, B. Lee, S. Saroiu, and V. Bahl. The Home Needs an Operating System (and an App Store). In ACM Hotnets IX, 2010.

[6] FitPC2. http://www.fit-pc.com/web/.

[7] Google Power Meter. http://www.google.com/powermeter/about/.
[8] Google Trend: Solar Homes. http://www.google.com/ trends?q=+solar+homes\&ctab=0\&geo=all\&date $=a l l$.

[9] H. Hu. Risk-Conscious Design of Off-Grid Solar Energy Houses. PhD dissertation, The Georgia Institute of Technology, Department of Architecture, Nov. 2009.

[10] Intel Home Energy Meter. http://edc.intel.com/ embedded/homeenergy/323157-001US.pdf.

[11] Kyocera Solar Panels. http://www.altersystems.com/catalog/ kyocera-kc120-120-watt-solar-panel-p-569.html.

[12] W. meters. https://www . wattsupmeters . com/secure/index.php.

[13] Outback FlexMax 60 Solar Charge Controller. http://www.altestore.com/store/ Charge-Controllers/Solar-Charge-Controllers/ MPPT-Solar-Charge-Controllers/ Outback-Solar-Charge-Controllers-MPPT/ Outback-Flexmax-60-Solar-Charge-Controller/ p6875/.

[14] Outback Mate Remote. http://www.altestore.com/store/Inverters/ Inverter-Accessories/Remote-Controls/ Outback-Mate-Remote-Monitor-And-Control/p857/.

[15] T. Schmid, D. Culler, and P. Dutta. Meter any wire, anywhere by virtualizing the voltage channel. In BuildSys, 2010.

[16] L. Sherwood. US Solar Market. 2010.

[17] J. Taneja, D. Culler, and P. Dutta. Towards cooperative grids: Sensor/actuator networks for promoting renewables. In SmarGridComm, 2010. 